\title{
Selection and application of peptide mimotopes of MPT64 protein in Mycobacterium tuberculosis
}

\author{
Hua Yang, Zhong-Hua Liu, Li-Ting Zhang, Jie Wang, Huan-Seng Yang, \\ Lian-Hua Qin, Rui-Liang Jin, Yong-Hong Feng, Zhen-Ling Cui, \\ Rui-Juan Zheng and Zhong-Yi Hu
}

Correspondence

Zhong-Yi Hu

shhuzy@163.com

Received 12 August 2010

Accepted 30 September 2010
Shanghai Key Laboratory of Tuberculosis, Shanghai Pulmonary Hospital, Tongji University School of Medicine, 507 Zhengmin Road, Shanghai 200433, PR China

\begin{abstract}
Antibody responses can be useful markers of tuberculosis (TB) infection, especially in the screening of extra-pulmonary TB. MPT64 is an important antigen in Mycobacterium tuberculosis (MTB) infection and is used in serological diagnosis. However, large variability in the diagnostic accuracy of MPT64 as a serological tool has limited its application. Phage-displayed random peptide libraries have emerged as a powerful technique to select peptides (epitopes) or mimotopes that may serve as surrogate diagnostic markers in serological tests. In the present study, this method was employed to identify mimotopes of the MPT64 protein of MTB by screening a linear heptapeptide library with rabbit antibodies raised against MPT64 protein. Two antigenic mimotopes (M2 and M6) resembling B-cell epitopes of MPT64 were identified that bound the affinity purified anti-MPT64 polyclonal antibodies and competed with MPT64 for antibody binding. From the results of sequence alignment and a structure modelling figure of MPT64, the sequence of the 2nd to 5 th amino acids (DSML) of M2 was totally consistent with the sequence of the 224th to 227th amino acids of MPT64 and the peptide is located on the surface of the space structure of MPT64, suggesting that it might be a linear epitope of MPT64. The recognition of both phage-displayed and synthetic peptides of M2 by the anti-MPT64 polyclonal antibodies also supported this. Although no recurring sequence and no analogue to MPT64 of M6 were found for sequence alignment, the recognition of both phage-displayed and synthetic peptides of M6 by the anti-MPT64 polyclonal antibodies indicated that it might be a mimotope of a conformational epitope of MPT64. According to the results of the reactivity of human sera with synthetic M2 and M6 peptides and MPT64, M2 showed a significantly higher AUC and sensitivity than M6 and MPT64, especially for the sera from sputum-negative TB patients, suggesting that the M2 mimotope may be useful in serological diagnostic testing for TB.
\end{abstract}

\section{INTRODUCTION}

With extraordinarily high rates of morbidity and mortality worldwide, tuberculosis (TB) remains one of the major global health problems. One third of the world's population is infected with Mycobacterium tuberculosis (MTB), the aetiological agent of $\mathrm{TB}$, which causes 1.1 to 1.7 million deaths per year (WHO, 2009). The current epidemiological situation has highlighted the importance of early diagnosis.

Antibody responses can be useful and rapid indicators of TB infection, especially in the rapid screening of extrapulmonary TB. Serological diagnosis is simple, rapid and requires only a single visit to the clinic, compared with

Abbreviations: AUC, area under the curve; BCG, Bacillus CalmetteGuérin; HRP, horseradish peroxidase; MTB, Mycobacterium tuberculosis; RDB-CMA, Respiratory Disease Branch of the Chinese Medical Association; ROC, receiver operating characteristic; TB, tuberculosis; $\mathrm{TMB}$, tetramethylbenzidine. isolation of the slow-growing MTB, which takes up to 3 or 4 weeks (Chan et al., 2000). MPT64 (Mycobacterium protein tuberculosis 64 ) is a $23 \mathrm{kDa}$ secreted protein and important protective antigen of MTB with a distinct possibility for specificity, as clearly demonstrated in immunohistochemistry studies of tuberculous lymphadenitis and pleuritis (Mustafa et al., 2006; Baba et al., 2008). The gene encoding this protein is only present in MTB, virulent Mycobacterium bovis and a few Bacillus CalmetteGuérin (BCG) vaccine strains, but is not found in most strains of BCG, such as Pasteur and Danish strains, which have been used as vaccine strains in China (Kamath et al., 1999). Patients with TB and healthy people vaccinated with BCG in China can be distinguished by using MPT64 as a rapid diagnostic antigen. However, a large variability in the diagnostic accuracy of MPT64 has been reported, depending on the recombinant antigen used in the assays (Silva et al., 2008). The accuracy may be influenced by the 
antigenic variability associated with protein purification or contamination derived from the cloning vectors used to produce recombinant protein (Baassi et al., 2009). Specific peptides synthesized according to the putative Bcell epitopes can permit the uniformity and standardization of antigen preparations, and potentially enhance the specificity by reducing cross-reactivity with other antigens (Liang et al., 1999). The B-cell epitopes of MPT64 have been mapped with $\mathrm{N}$-terminal and C-terminal deletion mutants, and Western blotting has revealed that one linear and three conformational epitopes, which were separately located within an approximately 30 residue amino acids sequence of MPT64, could bind to the five murine antibodies. However, the amino acid sequences of these epitopes remain unknown (Oettinger \& Andersen, 1994).

Though B-cell epitopes can be directly identified using biochemical or physical experiments, such as X-ray crystallography of antibody-antigen complexes, these experiments are costly, time-consuming and are not always successful (Gershoni et al., 2007). Computational methods to predict B-cell epitopes are much more efficient and cost effective. However, such computational methods are focused on the prediction of linear epitopes (Huang et al., 2008; Saha \& Raghava, 2006). In vitrosynthesized peptides have been widely applied for the mapping of B-cell epitopes, although it is not possible to detect epitopes that are conformationally dependent (Larsen et al., 2006). In the past decade, phage-display random peptide libraries have emerged as a powerful technique to select peptides (epitopes) or mimotopes, which mimic natural protein epitopes recognized by specific antibodies, and can thus serve as surrogate diagnostic markers in serological tests or play a vital role in the development of peptide vaccines (Scott et al., 1992). The antibody affinity-selected mimotopes can be selected by their capacity of binding to the antibodies directly against a given antigen (Dybwad et al., 1993). Mimotopes of several antigens of MTB have been selected successfully, such as lipoarabinomanan, Hsp16.3 and neutral polysaccharides (Gevorkian et al., 2005; Saha et al., 2005; Sharma et al., 2006). Peptide epitopes or mimotopes of known and unknown mycobacterial antigens can be isolated by using phage-display techniques, and peptides synthesized according to the mimotopes have shown potential for TB diagnostics and immunotherapy (Barenholz et al., 2007). In this study, the phage-display method was employed to identify mimotopes of the MPT64 protein of MTB by screening a linear heptapeptide library with rabbit antibodies raised against the MPT64 protein of MTB. A bioselection to identify phages displaying peptides specifically binding to the antiMPT64 antibody was performed, and the expressed peptides were analysed for their ability to mimic MPT64. Our results show that the screening resulted in the isolation of peptides mimicking the MPT64 protein of MTB at antigenic levels.

\section{METHODS}

Human sera. Venous blood was collected from 95 patients with confirmed pulmonary TB from Shanghai Pulmonary Hospital, Shanghai, PR China, before the start of treatment. Individual patients were diagnosed, according to the following criteria: (i) TB symptoms and signs, including cough, cough producing phlegm, coughing up blood, fever, night sweats, fatigue, loss of appetite, weight loss, chest pain, breathing difficulty, etc.; (ii) TB lesion on chest X-ray or computed tomography scan; (iii) strongly positive PPD (tuberculin pure protein derivative) skin test; (iv) the anti-TB treatment was effective; (v) positive smear or positive culture; (vi) bronchoscopy; and (vii) biopsy of the affected tissue and pathological examination. Diagnosis was made by satisfying any three of the criteria (i)-(iv) or any one of the criteria (v)(vii) (RDB-CMA, 2001). The acid-fast staining of bacilli smears and mycobacterial culture of sputa were performed in our laboratory, according to the Chinese Laboratory Science Procedure of Diagnostic Bacteriology in Tuberculosis (CAA, 1995). Among 95 patients, 39 were sputum-positive and the others were negative. Blood was also drawn from 85 healthy individuals who had been vaccinated with BCG during childhood. All patients and healthy controls in this study were negative for human immunodeficiency virus antibodies. This study was approved by the Research Ethics Committee of the Shanghai Pulmonary Hospital. Sera were separated according to the standard protocol and were stored in aliquots at $-70{ }^{\circ} \mathrm{C}$ until used.

MPT64 antigen and anti-MPT64 antibodies. Recombinant antigen MPT64 was purified as a His-tagged protein as described by Abe et al. (1999). For the generation of polyclonal antibodies against MPT64, four New Zealand white rabbits $(2-2.5 \mathrm{~kg})$ received subcutaneous injection of recombinant antigen MPT64 $(0.25 \mathrm{mg}$ in $0.5 \mathrm{ml}$ PBS emulsified with an equal volume of Freund's adjuvant). The animals received booster injections (on day 14, 24 and 34 after the initial priming immunizations) with $0.25 \mathrm{mg}$ MPT64 in $0.5 \mathrm{ml}$ PBS with $0.5 \mathrm{ml}$ incomplete Freund's adjuvant. The animals were killed by carotid exsanguinations on day 7 after the last immunization. The titre of the anti-MPT64 antiserum examined by ELISA was $1: 32000$. The polyclonal antibodies were purified by MPT64Sepharose column chromatography, and detected by SDS-PAGE and the Bradford method.

Phage-display selection and peptide sequencing. The PhD-7
phage-display library was purchased from New England Biolabs. The
complexity of the library was approximately $2.8 \times 10^{9}$ transformants.
Affinity purified polyclonal anti-MPT64 rabbit antibody was used for
affinity selection, according to the manufacturer's manual (http://
www.neb.com/nebecomm/products/productE8100.asp). After four
rounds, individual phage clones were isolated. The phage ssDNA
was purified and used for DNA sequencing, which was performed by
Sangon Biotech with the $-96 \mathrm{gIII}$ sequencing primer. The DNA and
amino acid sequences of the peptides were analysed and aligned by
computer with DNASTAR software (www.dnastar.com). Individual
phage clones with different sequences were amplified according to the
manufacturer's manual.

Reverse phage ELISA. To evaluate the binding of antibodies to the selected phage-displayed peptides, a 96-well ELISA plate was coated with $100 \mu \mathrm{g}$ affinity purified polyclonal anti-MPT64 rabbit antibody $\mathrm{ml}^{-1}$ in $100 \mu \mathrm{l}\left(0.1 \mathrm{~mol} \mathrm{l}^{-1}\right) \mathrm{NaHCO}_{3}$ buffer overnight at $4{ }^{\circ} \mathrm{C}$. After removing the coating buffer, $300 \mu$ l blocking buffer was added and the plate was incubated for at least $1 \mathrm{~h}$ at $4{ }^{\circ} \mathrm{C}$. Another plate without coating was incubated with blocking buffer too, for detection of the binding of BSA to the selected phage-displayed peptides. After washing with Tris-buffered saline, $0.5 \%$ Tween 20 (TBST) six times, phage particles were suspended in TBST and added to the wells at $10^{9}$ p.f.u.. After a $1-2 \mathrm{~h}$ incubation period with gentle shaking at room temperature, the plates were washed six times. Subsequently, capture 
of the phages by antibodies was detected with anti-M13 mAb conjugated to horseradish peroxidase (HRP) (GE Healthcare) using tetramethylbenzidine (TMB) solution as a substrate. The reaction was measured by reading the absorbance at 450 and $620 \mathrm{~nm}$ using an ELISA reader (MK3; Thermo). All samples were tested in triplicate.

Competitive ELISA. The affinity purified polyclonal anti-MPT64 rabbit antibody and MPT64 protein were used in the competitive inhibition ELISA to test the specificity of selected phage-peptide clones. The plates were coated with anti-MPT64 antibody and saturated with blocking buffer. After six washings with TBST, $50 \mu \mathrm{l}$ MPT64 protein at different concentrations $\left(0,0.2 \times 10^{-3}, 0.5 \times 10^{-3}\right.$, $\left.2 \times 10^{-3}, 5 \times 10^{-3}, 10 \times 10^{-3} \mathrm{~g}^{-1}\right)$ and $50 \mu \mathrm{l}$ phage particles at $10^{10}$ p.f.u. $\mathrm{ml}^{-1}$ were mixed and added to the wells, and incubated for $1 \mathrm{~h}$ with gentle shaking at room temperature. After washing six times, the ELISA procedure was performed as described above.

Peptide synthesis. For the derivation of synthetic peptides corresponding to the selected MPT64 mimotopes, a structurally flexible linker (GGGS) was added to the representative consensus sequences at the C-terminal ends to obtain the effective conformations of peptides. The desalted peptides M2 (SDSMLSWGGGS), M6 (FHTHISVGGGS) and M2C11 (VPRSAIDSMLA) were synthesized, purified and identified by Shanghai Bootech BioScience \& Technology.

Indirect ELISA. Indirect ELISA tests were performed by coating 96well ELISA plates overnight at $4{ }^{\circ} \mathrm{C}$ with $100 \mu$ per well of coating solution (17.5 mmol Na $\mathrm{CO}_{3} \mathrm{l}^{-1}, 32.5 \mathrm{mmol} \mathrm{NaHCO} \mathrm{H}^{-1}, 15 \mathrm{mmol}$ $\mathrm{MgCl}_{2} .6 \mathrm{H}_{2} \mathrm{Ol}^{-1}, \mathrm{pH}$ 9.6) containing either $10^{10}$ phages $\mathrm{ml}^{-1}, 1-32 \mu \mathrm{g}$ peptide $\mathrm{ml}^{-1}$ or $1 \mu \mathrm{g}$ MPT64 protein $\mathrm{ml}^{-1}$. Plates were washed three times with $0.02 \%$ Tween 20 in PBS (PBST) and blocked with $1.5 \%$ non-fat milk powder in PBS for $1 \mathrm{~h}$ at $37^{\circ} \mathrm{C}$. After washing three times with PBST, serum or polyclonal antibody dissolved in PBST (100 $\mu \mathrm{l}$ per well) was then added and the plates incubated for $1 \mathrm{~h}$ at $37^{\circ} \mathrm{C}$. Plates were then washed three times with PBST. HRP-labelled goat antihuman immunoglobulin or anti-rabbit immunoglobulin (Sigma) was added $(100 \mu \mathrm{l}$ per well $)$ and incubated for $1 \mathrm{~h}$ at $37^{\circ} \mathrm{C}$; this was followed by the addition of TMB substrate and measurement of the absorbance with an ELISA reader. All samples were tested in triplicate.

Statistics analysis. Test results were presented as means $\pm \mathrm{SD}$. The different groups were compared using the two-tailed, unpaired Student's $t$-test. $P$ values of $<0.05$ were considered to be significant. The receiver operating characteristic (ROC) curve was used to evaluate the performance of the ELISA tests with two categories (TB patients and healthy controls); the area under the curve (AUC) was used as a measure of diagnostic quality. The difference between the AUC was compared using the pair-wise comparison of ROC curves, $P$ values of $<0.05$ were considered to be significant.

\section{RESULTS AND DISCUSSION}

\section{Selection of phage clones and analysis of peptide sequences}

MPT64 has been shown to be specific for MTB complex organisms (Elhay et al., 1998), and has been evaluated in diagnostic tests (Harboe et al., 1986). With the purpose of identifying epitopes representing MTB MPT64, polyclonal antibodies directed against MPT64 were used as a target for biopanning. New Zealand rabbits were immunized with recombinant antigen MPT64 of MTB. After four immunizations, the rabbit sera showed a titre of $1: 32000$, as assessed by ELISA using MPT64 as antigen. Polyclonal antibodies were purified by MPT64-Sepharose column chromatography. The relative molecular mass of the antibody was about $50 \mathrm{kDa}$ as detected by SDS-PAGE, and the concentration was about $0.85 \mathrm{~g} \mathrm{l}^{-1}$ as detected by the Bradford method. Affinity purified polyclonal antiMPT64 rabbit antibody was used for the direct isolation of mimotopes of MPT64. After the fourth round of panning, the peptide-coding regions of randomly selected phage clones were sequenced. The results (Table 1) showed that recurring sequences could be detected, indicating their preferential binding with the antibody. A total of 16 of the 26 randomly selected phage clones belonged to a group (M2), in which all the clones had the same sequence (SDSMLSW). This may be the result of higher specificity or binding affinity to the target of the peptides (Scala et al., 1999). Sequence alignment to the amino acid sequence of MTB MPT64 showed the 2nd to 5th amino acids of the M2 sequence (DSML) were totally consistent with the 224th to 227th amino acids of the MPT64 sequence. According to the structure modelling figure of MPT64 (Fig. 1) analysed with PyMOL software (www.pymol.org), the amino acids sequence DSML is located on the surface of the space structure of MPT64, suggesting that the DSML may be a linear epitope of MPT64. Four other phage clones belonged to a group (M4) that had the same sequence (GPIDDAF), but no analogue was found for sequence alignment to the amino acids sequence of MPT64. Also, other phage clones did not have any consensus sequence to MPT64. An analogue was not found among eight amino acid sequences either.

\section{Binding affinity and specificity of the selected phage clones for anti-MPT64 antibodies}

The relative efficiency of binding of the selected recombinant phage clones to anti-MPT64 antibodies was assessed

Table 1. Anti-MPT64 antibody-binding peptide sequences selected after the fourth round of panning

A total of 26 individual phage clones were isolated and the phage ssDNA was purified and used for DNA sequencing. The DNA and amino acids sequences of peptides were analysed and aligned by computer with DNASTAR software. Eight peptide sequences and the number of times each sequence was independently isolated are given.

\begin{tabular}{|c|c|c|}
\hline Clone sequence identifier & $\begin{array}{l}\text { Amino acids } \\
\text { sequence }\end{array}$ & Frequency \\
\hline $\begin{array}{l}\text { M2, M3, M7-M12, M14, M15, } \\
\text { M16, M18, M19, M20, M24, M25 }\end{array}$ & 5 SDSMLSW & $16 / 26$ \\
\hline M4, M13, M17, M22 & GPIDDAF & $4 / 26$ \\
\hline M1 & HDASASL & $1 / 26$ \\
\hline M5 & TPTAPRQ & $1 / 26$ \\
\hline M6 & FHTHISV & $1 / 26$ \\
\hline M21 & HIRLSPA & $1 / 26$ \\
\hline M23 & SMPTYNK & $1 / 26$ \\
\hline M26 & HSSPTLS & $1 / 26$ \\
\hline
\end{tabular}




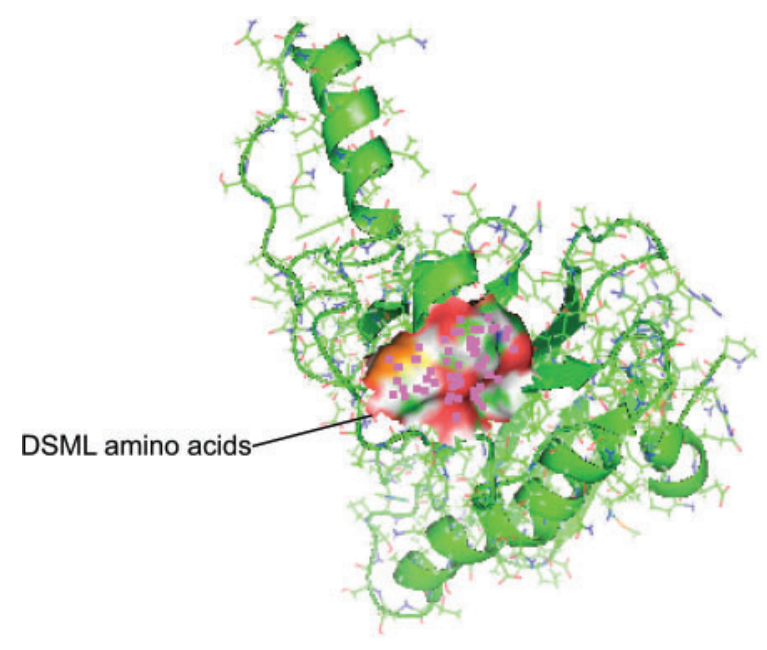

Fig. 1. Structure modelling figure of MPT64 protein and DSML amino acids. The structure modelling figure of MPT64 was analysed with PyMOL software (www.pymol.org), and the amino acids sequence DSML (shown in red) was located on the surface of the space structure of MPT64.

by reverse phage ELISA. M6 and M2 phage clones could bind to anti-MPT64 antibodies, and the reactivity of M6 was higher than that of M2 (data not shown). Competitive ELISAs confirmed that MPT64 protein could inhibit the binding of the M2 and M6 phage clones to anti-MPT64 antibodies, and the inhibition was concentration dependent, indicating the specificity of the observed phenomena (Fig. 2). When M2 and M6 phage clones were used as antigens in ELISA with human sera, a stronger reactivity was found with sera from sputum-positive TB patients $(n=32)$ compared with sera from healthy examination individuals $(n=31)$ (data not shown). Significance was determined against the negative-control group by the twotailed, unpaired Student's $t$-test $(P<0.0001)$.

\section{Binding properties of synthetic M2 and M6 peptides to polyclonal anti-MPT64 antibody}

To study the immunological properties of mimotopes in the form of phage-free molecules, peptides based on amino acids sequences of M2 and M6 phage clones were chemically synthesized. The motif-containing peptides were synthesized flanked by a spacer sequence GGGS on the $\mathrm{C}$ terminus, simulating the phage molecular context. To determine whether the M2 and M6 synthetic peptides were capable of binding polyclonal anti-MPT64 antibody, and the minimum binding concentration, we performed indirect ELISAs. To test the importance of the upstream and downstream amino acids of DSML, we designed a control peptides M2C11 (amino acids 218 to 228 of MPT64) and detected the binding properties of M2 and $\mathrm{M} 2 \mathrm{C} 11$. The plates were coated with $4 \mu \mathrm{g}$ peptide $\mathrm{ml}^{-1}$; polyclonal anti-MPT64 antibody double diluted in PBST

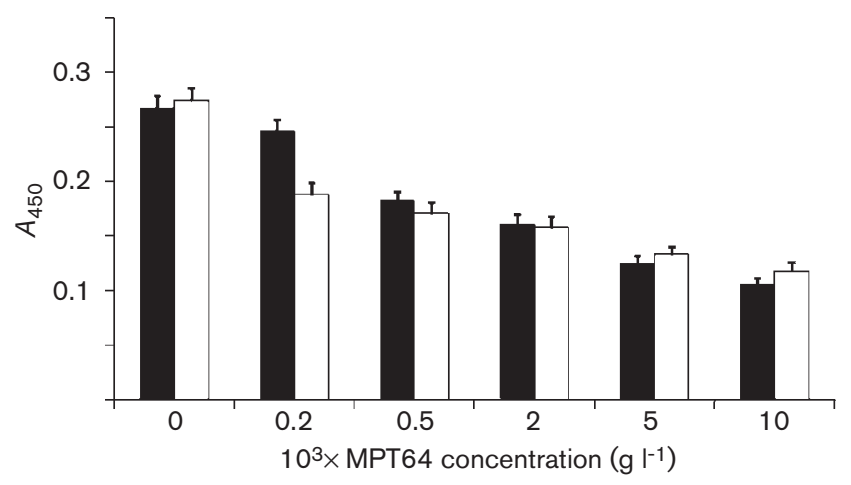

Fig. 2. Competitive binding of $M 2$ and $M 6$ phage clones against MPT64 by ELISA. The plates were coated with anti-MPT64 antibody and saturated with blocking buffer. After washing, $50 \mu \mathrm{l}$ MPT64 protein at different concentrations $\left(0,0.2 \times 10^{-3}\right.$, $0.5 \times 10^{-3}, 2 \times 10^{-3}, 5 \times 10^{-3}$, and $10 \times 10^{-3} \mathrm{~g} \mathrm{I}^{-1}$ ) and $50 \mu \mathrm{l}$ phage particles at $10^{10}$ p.f.u. $\mathrm{ml}^{-1}$ were mixed, added to the wells and incubated. Capture of the phages by antibodies was detected with anti-M13 mAb conjugated to HRP using TMB as a substrate. The reaction was measured by reading the absorbance at 450 and $620 \mathrm{~nm}$. The data represent the mean and SD of triplicate wells. The results confirmed that MPT64 protein could inhibit the binding of the M2 and M6 phage clones to anti-MPT64 antibodies and the inhibition was concentration-dependent. M2, Black bars; M6, white bars.

was then added and the plates incubated; the ELISA procedure was performed as described in Methods. From the results of indirect ELISAs (data not shown), we found that the binding properties of M2 were a little higher than $\mathrm{M} 2 \mathrm{C} 11\left(K_{\mathrm{d}}\right.$ value $4.9 \pm 0.48 \times 10^{-9}$ and $5.8 \pm 0.65 \times 10^{-9}$ mol $1^{-1}$ for M2 and M2C11, respectively), which suggested that the upstream and downstream amino acids of DSML of MPT64 may not be necessary for the binding to antibodies, or maybe the upstream and downstream amino acids of DSML of peptide M2 (S-DSML-SW) improve the binding properties of M2. The recognition of both phagedisplayed and synthetic peptide M2 by the anti-MPT64 polyclonal antibodies indicated that M2 peptide might be an antigenic mimotope resembling the B-cell epitopes of MPT64, and the DSML maybe a linear epitope of MPT64.

Although no recurring sequence and no analogue to MPT64 were found for sequence alignment, the recognition of both phage-displayed and synthetic peptide M6 by the anti-MPT64 polyclonal antibodies indicated that peptide M6 might be a mimotope of a conformational epitope of MPT64. It is not surprising that biopanning using polyclonal antibodies resulted in the isolation of different clones carrying peptide mimotopes of different epitopes. To confirm the binding properties of M6, we performed indirect ELISAs to detect the minimum binding concentration of synthetic peptide M6 (data not shown). Different from the result of ELISA of phage clones, the binding affinity of M6 $\left(K_{\mathrm{d}} 1.0 \pm 0.15 \times 10^{-8} \mathrm{~mol} \mathrm{l}^{-1}\right)$ is 


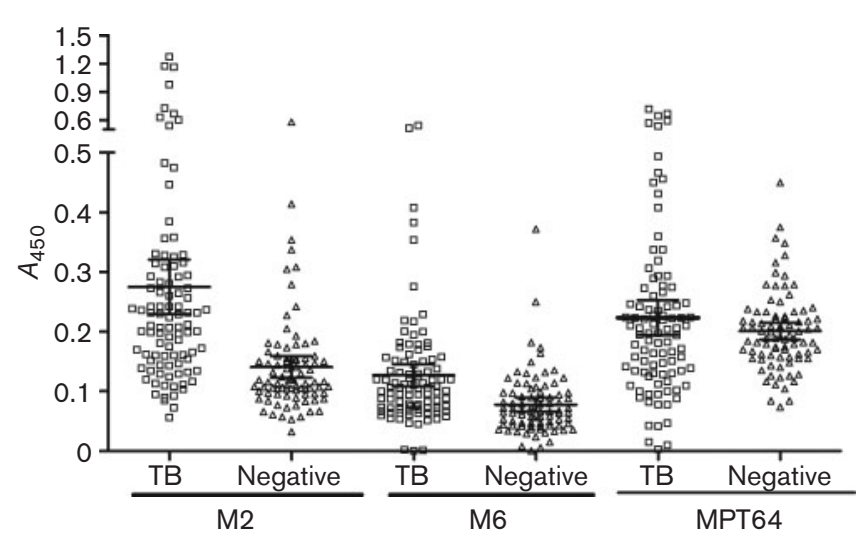

Fig. 3. Scatter dot plot representation of the distribution of levels of antibodies ( $\mathrm{gGG}$ ) to the peptides M2 and M6, and MPT64 protein in TB patients $(\square)$ and negative controls $(\triangle)$. The plates were coated with $4 \mu \mathrm{g}$ peptide $\mathrm{M} 2 / \mathrm{M} 6 \mathrm{ml}^{-1}$ or $1 \mu \mathrm{g} \mathrm{MPT64}$ protein $\mathrm{ml}^{-1}$ per well, as described in Methods. Sera from TB patients $(n=95)$ and healthy individuals $(n=85)$ were tested. The $95 \%$ confidence interval for the mean is represented by error bars. The results are shown as the mean $A_{450}$ value of each triplicate sample read $20 \mathrm{~min}$ after the addition of substrate. Significance was determined against the negative control groups for $\mathrm{M} 2$ and M6 peptides $(P<0.0001)$, while the titres of MPT64-binding antibodies in the sera of TB patients were not significantly higher than those of the sera of healthy individuals $(P=0.1878)$.

lower than M2 ( $\left.K_{\mathrm{d}} 4.9 \pm 0.48 \times 10^{-9} \mathrm{~mol} \mathrm{l^{-1 }}\right)$, which suggested the instability of the binding to antibodies.

\section{Reactivity of human sera with synthetic M2 and M6 peptides}

To evaluate the reactivity of synthetic M2 and M6 peptides to human sera, we used an ELISA to test whether the sera of TB patients contain antibodies to MPT64 that can also bind M2 and M6 peptides. M2/M6 peptides and MPT64 were coated on 96-well ELISA plates and sera samples of 95 sputum-positive or -negative TB patients and 85 healthy individuals were verified using indirect ELISA. From the
ELISA, we found significantly higher titres of M2 and M6 peptides-binding antibodies in the sera of TB patients compared with the sera of healthy individuals $(P<0.0001$; Fig. 3), while the titres of MPT64-binding antibodies in the sera of TB patients were not significantly higher than those of the sera of healthy individuals ( $P=0.1878$, Fig. 3). But for 39 sputum-positive TB patients, the compared results of M2/M6 peptides and MPT64 were all significant, $P<0.0001, P<0.0001$ and $P=0.0017$ (data not shown). For 56 sputum-negative TB patients, the compared results of M2/M6 peptides were also significant, $P<0.0001$ and $P=0.0034$, but for MPT64, they were not, $P=0.4195$ (figure not shown). Table 2 shows the data of the AUC of M2 and M6 peptides and MPT64 and the difference between the AUC. The difference between the areas of M2 and M6, M2 and MPT64, and M6 and MPT64 were all significant for all TB patients and for the 39 sputumpositive ones. For 56 sputum-negative TB patients, the difference between the areas of M2 and MPT64, M6 and MPT64 were also significant, but for M2 and M6, they were not (Table 2). When the cut-off point for the $A_{450}$ value was 0.17 , the results of the ELISA based on peptide M2 showed a specificity of $80.0 \%$, the same as M6 and MPT64, while the sensitivity of M2 reacted at $66.3 \%$, significantly higher than M6 (47.37\%, P=0.008) and MPT64 (32.6\%, $P<0.0001)$ with $\chi^{2}$ tests. For 39 sputum-positive TB patients, when the specificity was $80.0 \%$, the sensitivities of M2, M6 and MPT64 were 69.2, 59.0 and $48.7 \%$, respectively; the difference was not significant $(P=0.183)$. But for 56 sputum-negative samples, the sensitivity of M2 $(66.1 \%)$ was significantly higher than M6 (39.3\%, $P=0.005)$ and MPT64 $(21.4 \%, P<0.0001)$. The results indicated the possibility of using the M2 mimotope for the serological diagnosis of TB, especially for sputum-negative patients.

Compared with the commonly used recombinant protein in TB diagnostic tests, peptides have many benefits. The synthetic peptides can be inexpensive to produce, welldefined and highly reproducible (Shin et al., 2002); while purifying recombinant protein from $E$. coli is a complicated procedure (Chiang et al., 1997). In addition, recombinant protein from different batches may differ in antigenic

Table 2. AUC for the ROC curve of M2 and M6 peptides, and MPT64, and the difference between the AUC values

M2 and M6 peptides, and MPT64 were coated on the 96-well ELISA plates and sera samples of 95 TB patients (39 sputum positive and 56 sputum negative) and 85 healthy individuals were verified using indirect ELISA. The ROC curve was used to evaluate the performance of the ELISA tests; the AUC was used as a measure of diagnostic quality. The difference between the AUC values was compared using the pair-wise comparison of ROC curves. $P$ values of $<0.05$ were considered to be significant.

\begin{tabular}{|c|c|c|c|c|c|c|}
\hline \multirow[t]{2}{*}{ Type of TB patients $(n)$} & \multicolumn{3}{|c|}{ AUC } & \multicolumn{3}{|c|}{ Difference between the AUC values } \\
\hline & M2 & M6 & MPT64 & M2 and M6 & M2 and MPT64 & M6 and MPT64 \\
\hline Sputum-positive (39) & 0.846 & 0.756 & 0.603 & $\mathrm{Z}=2.305, P=0.021$ & $Z=4.775, P<0.001$ & $\mathrm{Z}=2.801, P=0.005$ \\
\hline Sputum-negative (56) & 0.760 & 0.705 & 0.569 & $\mathrm{Z}=1.131, P=0.258$ & $\mathrm{Z}=3.319, P=0.001$ & $\mathrm{Z}=2.412, P=0.016$ \\
\hline
\end{tabular}


properties, leading to variability in diagnostic accuracy (Harboe et al., 1992). Thus, we draw the conclusion that the M2 peptide may be an efficient and promising mimotope of MPT64 and shows potential to substitute MPT64 and be used as an antigen in the serological diagnosis of TB.

\section{ACKNOWLEDGEMENTS}

This work was supported by the Ministry of Science and Technology of the People's Republic of China (grant no. 2008ZX10003-003-02), and the Science and Technology Commission of Shanghai Municipality, Shanghai, PR China (grant no. 09ZR1425800).

\section{REFERENCES}

Abe, C., Hirano, K. \& Tomiyam, T. (1999). Simple and rapid identification of the Mycobacterium tuberculosis complex by immunochromatographic assay using anti-MPB64 monoclonal antibodies. J Clin Microbiol 37, 3693-3697.

Baassi, L., Sadki, K., Seghrouchni, F., Contini, S., Cherki, W., Nagelkerke, N., Benjouad, A., Saltini, C., Colizzi, V. \& other authors (2009). Evaluation of a multi-antigen test based on B-cell epitope peptides for the serodiagnosis of pulmonary tuberculosis. Int J Tuberc Lung Dis 13, 848-854.

Baba, K., Dyrhol-Riise, A. M., Sviland, L., Langeland, N., Hoosen, A. A., Wiker, H. G. \& Mustafa, T. (2008). Rapid and specific diagnosis of tuberculous pleuritis with immunohistochemistry by detecting Mycobacterium tuberculosis complex specific antigen MPT64 in patients from a HIV endemic area. Appl Immunohistochem Mol Morphol 16, 554-561.

Barenholz, A., Hovav, A. H., Fishman, Y., Rahav, G., Gershoni, J. M. \& Bercovier, H. (2007). A peptides mimetic of the mycobacterial mannosylated lipoarabinomannan: characterization and potential applications. J Med Microbiol 56, 579-586.

CAA (1995). Chinese Laboratory Science Procedure of Diagnostic Bacteriology in Tuberculosis, pp. 9-21. Beijing: Chinese Antituberculosis Association (in Chinese).

Chan, E. D., Heifets, L. \& Iseman, M. D. (2000). Immunologic diagnosis of tuberculosis: a review. Tuber Lung Dis 80, 131-140.

Chiang, I.-H., Suo, J., Bai, K.-J., Lin, T.-P., Luh, K.-T., Yu, C.-J. \& Yang, P.-C. (1997). Serodiagnosis of tuberculosis. A study comparing three specific mycobacterial antigens. Am J Respir Crit Care Med 156, $906-$ 911.

Dybwad, A., Forre, O., Kjeldsen-Kragh, J., Natvig, J. B. \& Sioud, M. (1993). Identification of new B cell epitopes in the sera of rheumatoid arthritis patients using a random nanopeptides phage library. Eur $J$ Immunol 23, 3189-3193.

Elhay, M. J., Oettinger, T. \& Andersen, P. (1998). Delayed-type hypersensitivity responses to ESAT-6 and MPT64 from Mycobacterium tuberculosis in the guinea pig. Infect Immun $\mathbf{6 6}$, 3454-3456.

Gershoni, J. M., Roitburd-Berman, A., Siman-Tov, D. D., Tarnovitski, F. N. \& Weiss, Y. (2007). Epitope mapping: the first step in developing epitope-based vaccines. BioDrugs 21, 145-156.

Gevorkian, G., Segura, E., Acero, G., Palma, J. P., Espitia, C. Manoutcharian, K. \& López-Marín, L. M. (2005). Peptides mimotopes of Mycobacterium tuberculosis carbohydrate immunodeterminants. Biochem J 387, 411-417.
Harboe, M., Nagai, S., Patarroyo, M. E., Torres, M. L., Ramirez, C. \& Cruz, N. (1986). Properties of proteins MPB64, MPB70, and MPB80 of Mycobacterium bovis BCG. Infect Immun 52, 293-302.

Harboe, M., Wiker, H. G. \& Nagai, S. (1992). Protein antigens of mycobacteria studied by quantitative immunologic techniques. Clin Infect Dis 14, 313-319.

Huang, Y. X., Bao, Y. L., Guo, S. Y., Wang, Y., Zhou, C. G. \& Li, Y. X. (2008). Pep-3D-Search: a method for B-cell epitope prediction based on mimotope analysis. BMC Bioinformatics 9, 538.

Kamath, A. T., Feng, C. G., Macdonald, M., Briscoe, H. \& Britton, W. J. (1999). Differential protective efficacy of DNA vaccines expressing secreted proteins of Mycobacterium tuberculosis. Infect Immun 67, 1702-1707.

Larsen, J. E., Lund, O. \& Nielsen, M. (2006). Improved method for predicting linear B-cell epitopes. Immunome Res 2, 2.

Liang, F. T., Steere, A. C., Marques, A. R., Johnson, B. J., Miller, J. N. \& Philipp, M. T. (1999). Sensitive and specific serodiagnosis of Lyme disease by enzyme-linked immunosorbent assay with a peptides based on an immunodominant conserved region of Borrelia burgdorferi VlsE. J Clin Microbiol 37, 3990-3996.

Mustafa, T., Wiker, H. G., Mfinanga, S. G., Mørkve, O. \& Sviland, L. (2006). Immunohistochemistry using a Mycobacterium tuberculosis complex specific antibody for improved diagnosis of tuberculous lymphadenitis. Mod Pathol 19, 1606-1614.

Oettinger, T. \& Andersen, A. B. (1994). Cloning and B-cell-epitope mapping of MPT64 from Mycobacterium tuberculosis H37Rv. Infect Immun 62, 2058-2064.

RDB-CMA (2001). The guide for diagnosis and treatment of pulmonary tuberculosis. J Chin Tuber Respir Dis 24, 70-74 (in Chinese).

Saha, S. \& Raghava, G. P. (2006). Prediction of continuous B-cell epitopes in an antigen using recurrent neural network. Proteins 65, 40-48.

Saha, A., Sharma, A., Dhar, A., Bhattacharyya, B., Roy, S. \& Das Gupta, S. K. (2005). Antagonists of Hsp16.3, a low-molecular-weight mycobacterial chaperone and virulence factor, derived from phagedisplayed peptides libraries. Appl Environ Microbiol 71, 7334-7344.

Scala, G., Chen, X., Liu, W., Telles, J. N., Cohen, O. J., Vaccarezza, M., Igarashi, T. \& Fauci, A. S. (1999). Selection of HIV-specific immunogenic epitopes by screening random peptides libraries with HIV-1-positive sera. J Immunol 162, 6155-6161.

Scott, J. K., Loganathan, D., Easley, R. B., Gong, X. \& Goldstein, I. J. (1992). A family of concanavalin A-binding peptides from a hexapeptides epitope library. Proc Natl Acad Sci U S A 89, 5398-5402.

Sharma, A., Saha, A., Bhattacharjee, S., Majumdar, S. \& Das Gupta, S. K. (2006). Specific and randomly derived immunoactive peptides mimotopes of mycobacterial antigens. Clin Vaccine Immunol 13, 1143-1154.

Shin, J. S., Yu, J., Lin, J., Zhong, L., Bren, K. L. \& Nahm, M. H. (2002). Peptides mimotopes of pneumococcal capsular polysaccharide of $6 \mathrm{~B}$ serotype: a peptides mimotope can bind to two unrelated antibodies. J Immunol 168, 6273-6278.

Silva, V. M., Sardella, I. G., Luiz, R. R., Cunha, A. J., Cavalcanti, A. H., Mahavir, S., Barreto, M. M., Rodrigues, R. S., Carvalho, T. F. \& Saad, M. H. (2008). Immunoreactivity of five antigens of Mycobacterium tuberculosis in patients attending a public health care facility in an area with high endemicity for TB. Microbiol Immunol 52, 544-550.

WHO (2009). Global tuberculosis control. A short update to the 2009 report. WHO/HTM/TB/2009.426. Geneva: World Health Organization. 